\title{
Nicotinic receptor channelopathies and epilepsy
}

\author{
Ortrud K. Steinlein • Daniel Bertrand
}

Received: 3 November 2009 /Revised: 23 November 2009/Accepted: 24 November 2009 /Published online: 17 December 2009

(C) Springer-Verlag 2009

\begin{abstract}
Characterized by sudden episodes called seizures, epilepsy was recognized long ago as a neurological disorder that can have multiple forms ranging from benign to life threatening depending upon its severity. Although several evidences indicated that genes play an important role in at least half of the patients, it is only with the advances in molecular biology and genetics that the puzzle about oligogenic and monogenic epilepsies slowly starts to unfold. The finding of an association between a monogenic form of epilepsy and a mutation in the gene encoding the neuronal nicotinic acetylcholine receptor subunit CHRNA4 marked, in 1995, a turning point in our understanding of epilepsy. It also marked the first step towards the today widely acknowledged concept of epilepsies as channelopathies. Several mutations in nicotinic acetylcholine receptor genes have, since then, been identified, and the functional properties of these mutated receptors were characterized. In this work, we review, in the light of the latest discoveries, the effects caused by the mutations on the physiological properties of the receptors and the impact of such mutations on neuronal network functions.
\end{abstract}

Keywords Acetylcholine · Brain · Epilepsy ·

Neurotransmission $\cdot$ Nicotinic receptor

O. K. Steinlein

Institute of Human Genetics, University Hospital,

Ludwig Maximilians University,

Munich, Germany

D. Bertrand $(\square)$

Department of Neuroscience, Medical Faculty,

University of Geneva,

Geneva, Switzerland

e-mail: daniel.bertrand@unige.ch

\section{Introduction}

Already recognized as a brain disease by the Egyptians more than 3,000 years ago, epilepsy is a rather common neurobiological disorder that can be profoundly debilitating. Characterized by sudden recurrent episodes termed seizure, epilepsy ranges from benign to life threatening depending on the severity and frequency of the seizure episodes. While there is a general agreement that epilepsy is caused by hyper synchrony of brain neuron firing, epilepsy can have multiple origins and aspects depending upon the affected brain areas. The average prevalence rate of epilepsy is about 1:100-200, and most epilepsies are assumed to be caused by a combination of acquired and genetic factors, many of them still unknown. Predominantly genetic epilepsies constitute only a minority of all seizure disorders but are of major interest for science. Most of these monogenic epilepsies have prevalence rates of 1:100,000 or less, but the apparently straightforward relationship between an underlying mutation and the clinical phenotype nevertheless made them a firstchoice tool to study the basic principles of epileptogenesis at a molecular level. However, it is now acknowledged that even in the apparently simple monogenic epilepsies, the discovery of an underlying mutation not necessarily easily reveals the pathophysiological pathway that leads from this specific mutation to the clinically visible seizure. The complexity of the mutation-phenotype relationship is best demonstrated by the autosomal dominant nocturnal frontal lobe epilepsy (ADNFLE), a rare seizure disorder that has been first described about 15 years ago and studied intensively since then. It would be difficult to find another genetic disorder in which the known mutations are restricted to such a small part of the gene but cause the same degree of functional and clinical heterogeneity [14, 33, 37, 41, 48, 49]. ADNFLE not only was the first human epilepsy for which a mutation had 
been described [49] but also allowed the first glimpse into what turned out to be a major pathophysiological principle in epileptogenesis. Caused by mutations in different subunits of the neuronal nicotinic acetylcholine receptor (nAChR), ADNFLE was the first seizure disorder for which the importance of ion channels could be demonstrated. Other monogenic epilepsies followed, establishing the concept of epilepsies as channelopathies. Although some non-ion channel mutations have been described recently, the channelopathy hypothesis remains the major pathogenic concept in epilepsy research.

\section{Autosomal dominant nocturnal frontal lobe epilepsy}

The nocturnal clusters of brief motor seizures that are typical for ADNFLE occur mostly out of non-rapid eye movement sleep. Seizures are characterized by stereotyped movements that can be preceded by an aura phenomenon including epigastrial, sensory, or psychic symptoms. In some patients, sleepwalking periods are common in which they might move around and talk unintelligibly. Typically, seizures start with gasps, grunts, or vocalizations followed by thrashing hyperkinetic activity or tonic stiffening of the limbs and superimposed clonic jerking. Patients are often not aware of their seizures or they underestimate their number of seizures per night. The clustering of seizures can cause poor sleep quality, resulting in daytime fatigue. Treatment with antiepileptic drugs such as carbamazepine is often effective but in some patients fails to reduce seizure frequency sufficiently. The seizures often start in the second decade of life, but the age of onset can show considerable intra- and interfamilial variation (first to sixth decade of life). The seizure frequency tends to decline in middle age, and long-lasting or complete remissions are sometimes reported. Certain families display a pattern of clinical symptoms that not only includes epileptic seizures but also additional neurological features such as psychiatric disorders, mental retardation, or cognitive dysfunction $[4,11$, 35]. Most family's display an autosomal dominant pattern of inheritance with reduced penetrance, less commonly, the syndrome is caused by de novo mutations [41]. All but one of the known mutations was found in either CHRNA4 or CHRNB2, the genes coding for the $\alpha 4$ - and $\beta 2$-subunits of the nAChR, respectively. An additional mutation was described for the CHRNA2 gene in a family with a form of sleep-related epilepsy that resembles ADNFLE [2].

\section{Nicotinic acetylcholine receptors}

First identified at the neuromuscular junction, nAChRs belong to the large family of ligand-gated channels. Used for a long time as a model of synaptic transmission, the neuromuscular junction provided many clues about the basic principles underlying chemical neurotransmission that can be summarized as follows. Release of the neurotransmitter in the synaptic cleft causes a brisk increase in neurotransmitter concentration, which passively diffuses through the extremely narrow extracellular space of the synaptic cleft. Binding of the neurotransmitter on the receptor causes the opening of ionic pores in the postsynaptic membrane leading to the depolarization of the muscle fiber up to its firing threshold. This mechanism provokes a huge amplification factor allowing the propagation of the nerve impulse from the motoneuron termination to the very large muscle fiber. Although the basic principles of the neurotransmission identified at the neuromuscular junction provide a good framework for the functioning of brain synapses, multiple differences in the functioning and properties must also be considered when examining the neurotransmission in the central nervous system.

The very high density and almost pseudocrystalline state of nAChRs found in the Torpedo electro plaques provided the first images with electron microscopic resolution of an $\mathrm{nAChR}$ inserted in the postsynaptic membrane. Progresses made from the early negative staining with high-resolution microscopy yielded close to atomic resolution of the receptor structure [52]. To date, however, the best models yielding a resolution at near $2 \AA$ come from the acetylcholine binding protein for the extracellular domain and from a cationic bacterial channel $[8,26]$. Whilst data have been collected from different species, they all point out to the overall structure. Receptors result from the assembly of five subunits arranged in a donut-like manner around an axis of pseudosymmetry. Each subunit spans the membrane four times with its $\mathrm{N}$ - and C-terminal ends facing the extracellular domain. The extracellular domains are arranged mainly in $\beta$-sheet, and the ligand-binding pocket is formed at the interface between adjoining subunits. Subunits presenting two adjacent cysteines in their extracellular domain are termed $\alpha$, whereas other subunits are termed $\beta$ (or $\gamma, \delta$, and $\varepsilon$ in the case of the muscle receptors). The $\alpha$ subunits contribute to the principal domain of the ligand-binding site, whereas $\beta$ subunits contribute to the complementary site. Located in the center of such a pentamer, the ionic pore is bordered by the second transmembrane segments comparable to the staves of a barrel. The ionic selectivity and properties of the pore are therefore governed by the amino acids facing this central pore.

To date, 17 genes encoding for the muscle and neuronal nAChRs have been identified and cloned in the mammalian genome $[1,13]$. The high degree of homology observed across species together with the ancient age of nAChRs and the diversity of subunits gained during evolution strongly 
indicate an important contribution to brain function. By convention, genes encoding for the $\mathrm{nAChR} \alpha$ subunits are termed CHRNA1-10, whereas those encoding for $\beta$ subunits are termed CHRNB1-4.

The simplest form of an nAChR is composed of five identical subunits, such as in the homomeric $\alpha 7$ receptors. Additional complexities in which two or more subunit types are assembled in the same receptor are, however, widely observed in the central and peripheral nervous system. A prototype of such a receptor is the $\alpha 4 \beta 2$ which results from the assembly of $\alpha 4$ and $\beta 2$ subunits. While elegant experiments initially suggested that a functional receptor comprises two $\alpha 4$ and three $\beta 2$ subunits [12], more recent data suggest that $\alpha 4 \beta 2$ nAChRs may exist in different stoichiometries $[9,38,56]$. As additional alternative $\alpha$ or $\beta$ subunits can be incorporated into such a receptor structure, this gives rise to a large variety of receptor subtypes.

Although studies of native nAChRs became possible with intracellular recordings in brain slices, most of the physiological and pharmacological characterization of these receptors was obtained by recombinant expression studies. Important points demonstrated by these studies are the possibility to examine the receptor properties in isolation and to express human receptors as well as those from any other species. Characterization of the $\alpha 7$ receptors revealed that they are activated by high micromolar ranges of acetylcholine (ACh) and display a very fast desensitization during agonist exposure [23]. Another functional particularity of the $\alpha 7$ receptor is its high calcium permeability that was documented both in reconstituted and native receptors $[5,19,20,22,46]$. By comparison, the heteromeric $\alpha 4 \beta 2$ nAChRs display a higher sensitivity to ACh, slower desensitization, and lower calcium permeability [10, 45]. In addition, the $\alpha 4 \beta 2$ nAChRs show a dual phase in the concentration activation curve [9]. As a consequence, the receptor population can be divided in high and low affinity ion channels, and effects of the ligand will depend upon the fraction of receptors in either of these two states. Interestingly, prolonged exposure to nicotine was shown to change the ratio between high and low affinity indicating that receptor properties and their apparent affinity for the ligand can be modulated causing a change in the dynamic range of the receptor responses [9]. Moreover, the presence of high and low affinity components extents the dynamic range in which the receptor responds to ACh. For the human $\alpha 4 \beta 2$ receptors, the high affinity component displays an $\mathrm{EC}_{50}$ (the concentration causing the activation of half of the high affinity receptor population) at about $3 \mu \mathrm{M}$, whereas the $\mathrm{EC}_{50}$ for the low affinity is at about $60 \mu \mathrm{M}$. By consequence, the $\alpha 4 \beta 2$ receptor will respond to $\mathrm{ACh}$ concentrations ranging from 0.1 to $300 \mu \mathrm{M}$ or about three to four orders of magnitude. By comparison, a receptor such as $\alpha 7$ displays a dynamic range of about two orders of magnitude.
A biophysical characteristic shared by all neuronal nAChRs, but not the muscle receptor, is a strong inward rectification. The rectification is a (I-V) non-linear relationship between transmembrane potential $(\mathrm{V})$ and the current flowing through the receptors (I). For transmembrane potentials more negative than $-40 \mathrm{mV}$, a current flows through the receptors when they are activated, whereas the current is profoundly reduced when the membrane is less negative [3]. Rectification reflects the relationship between the probability of channel opening and the voltage, whereas the single channel conductance follows Ohms law [10]. Structure function studies revealed that rectification is attributable to the charged amino acid at the inner mouth of the ionic pore, as substitution of this charge renders the I$\mathrm{V}$ curve linear [18]. The high degree of conservation of this biophysical property across receptor subtypes and different animal species suggests a relevant functional phenomenon. The importance of the rectification is easily understood when considering the relationship between neurotransmitter release and the transmembrane potential of the postsynaptic neuron. Presence of acetylcholine will cause an inward current in the receptors only if the cell membrane potential is below $-40 \mathrm{mV}$. Thus, activation of neuronal $\mathrm{nAChRs}$ will have no significant effect if the cell is depolarized. Altogether, this mechanism can be considered as a coincidence detector in which the two conditions of presence of the neurotransmitter and sufficiently negative cell membrane potential must be fulfilled to elicit a functional response.

Studies carried out in brain or spinal cord slices revealed that a single neuron often expresses multiple receptor subtypes that can be classified according to their physiological and pharmacological properties (reviewed in [1]). Importantly, however, receptors are not homogenously distributed on the cell surface but are targeted to specific cell locations. Targeting to subcellular compartments depends upon multiple mechanisms in which the large intracellular loop formed between the third and fourth transmembrane segment plays an important role $[54,55]$. Such discrete receptor expression has fundamental consequences for the physiological role of nAChRs. For example, it is readily understood that receptors expressed on the cell soma will influence the cell resting potential and thereby its firing threshold and frequency of discharges. By opposition, receptors expressed on presynaptic boutons will have no influence on the cell resting potential but can regulate neurotransmitter release (reviewed in [13]). $\alpha 7$ nAChRs expressed on presynaptic endings have been shown to regulate the intracellular calcium concentration in these restricted subcellular compartments and modulate the release of neurotransmitter contained in the vesicular pool. Contrary to this, the $\alpha 4 \beta 2 \mathrm{nAChRs}$ expressed on presynaptic nerve endings were shown to cause a local 
membrane depolarization which activates the voltagedependent calcium channels and, indirectly, modulates neurotransmitter release. Albeit the functional outcomes of homomeric and heteromeric nAChRs resemble each other, their mechanisms of action clearly differ.

\section{Physiological properties of mutated channels}

Structure function studies of the nAChRs became possible with the isolation of cDNAs encoding for the different nAChRs subunits and their expression in host systems such as Xenopus oocytes or cell lines [3, 39]. These pioneer studies marked the first step towards the investigation of human neuronal nAChRs and the influence of mutations on the receptor function. Studies carried out with several nAChR subtypes highlighted the role of the second transmembrane domain, confirming the earlier hypothesis that this domain borders the ionic pore. Insertion of a single amino acid at the inner mouth of this transmembrane segment was shown to be sufficient to convert the ionic selectivity from cationic to anionic [21]. Largely confirmed in further studies, this initial finding illustrated the similarities in the structural organization and receptor function amongst the family of cys-loop receptors, which include the $\mathrm{GABA}_{\mathrm{A}}$, glycine, and $5 \mathrm{HT}_{3}$ receptors. A single amino acid exchange in this second transmembrane domain caused obvious changes in receptor desensitization and was interpreted as reflecting a modification in the gating of the desensitized state [44].

The identification in 1995 of a first ADNFLE mutation in CHRNA4, the gene encoding for the $\alpha 4 \mathrm{nAChR}$ subunit marked an important step in our understanding of the relevance of nAChRs in brain functions [49]. Affected individuals from this family showed a missense mutation causing the substitution of serine at location 248 by a phenylalanine. As this mutation is in the second transmembrane domain, it was predicted that it might affect the receptor properties. Functional studies carried out by expression of this variant and the control $\beta 2$ subunit indeed confirmed that this mutation alters the receptor properties [53]. While this mutation affects several properties of the receptor, such as desensitization, and calcium permeability, the relationship between this mutation and seizure susceptibility is not immediately obvious $[15,32]$. An interesting clinical observation was that seizures and dyskinesia in these patients were significantly reduced by the antiepileptic drug carbamazepine. Based on this observation, functional studies showed that the tricyclic compound carbamazepine preferentially inhibits the mutated S248F $\alpha 4$ containing receptors suggesting a correlation with the clinical finding [42]. As initial experiments were carried out by expression of the mutated $\alpha 4$ with $\beta 2$, the experimental conditions were not reflecting the patient's heterozygous conditions with respect to the mutation. To get one step closer to the physiological conditions, it was necessary to examine if expression of a mutated $\alpha 4$ subunit shows dominant effects when co-expressed with the wild type $\alpha 4$ and $\beta 2$. Experiments carried out first in oocytes and then in cell lines established the dominant nature of the S248F mutant confirming that a single mutation can substantially alter receptor function $[6,7,28]$.

From the basic nature of the nAChRs, it was predicted that mutations in either the gene encoding for the $\alpha 4$ subunit or the gene encoding for the $\beta 2$ subunit could cause identical changes in receptor function. Whilst at first, only mutations in CHRNA4, which encodes the $\alpha 4$ subunit, were identified, subsequent studies also discovered mutations in CHRNB2, which encodes the $\beta 2$ subunit $[14,40]$. To date, six mutations in CHRNA4 and five in CHRNB2 have been found to be associated with ADNFLE. Providing a strong support for the causality between the high affinity $\alpha 4 \beta 2$ $\mathrm{nAChR}$ and a specific form of epilepsy, these mutations allow to search for a common alteration in receptor properties that is responsible for seizure triggering. Functional studies carried out in Xenopus oocytes with the various ADNFLE mutations revealed their dominant contribution when co-expressed with the control $\alpha 4$ and $\beta 2$ subunits and demonstrated that they all cause a gain of function effect [4, 28, 30, 47]. Altogether, it was concluded that either an increased acetylcholine sensitivity or a shift in the proportion between low and high-sensitivity receptors is the common trait linking together all mutations so far identified in CHRNA4 or CHRNB2. Typical effects on the acetylcholine concentration activation curve are shown in the upper panel of Fig. 1.

While presenting the first evidences for the causality between a mutation in a ligand-gated channel and epileptic seizures, these studies also pointed out the naturally occurring sequence variants that can be observed in many, if not all, genes. As most nAChR subtypes are expressed in the brain and the spinal cord, it would be of value to examine the possible relationship between gene variants and neurological disorders. The finding of an additional form of nocturnal epilepsy that is caused by a mutation in CHRNA2, the gene encoding for the $\alpha 2 \mathrm{nAChR}$ subunit, further support the hypothesis that additional, so far unknown, neurological disorders exist that are associated with nAChRs. First identified in an Italian family, the CHRNA2 mutation (I279N) was shown to cause an increased sensitivity to acetylcholine [2]. In this initial study, however, functional nAChRs were obtained only by expression of the $\alpha 2$ subunit with the $\beta 4$ subunit and therefore differed from the native receptors that most likely mainly result from the assembly of $\alpha 2$ with $\beta 2$. Further studies carried out with the $1279 \mathrm{~N} \alpha 2$ mutant expressed with the 

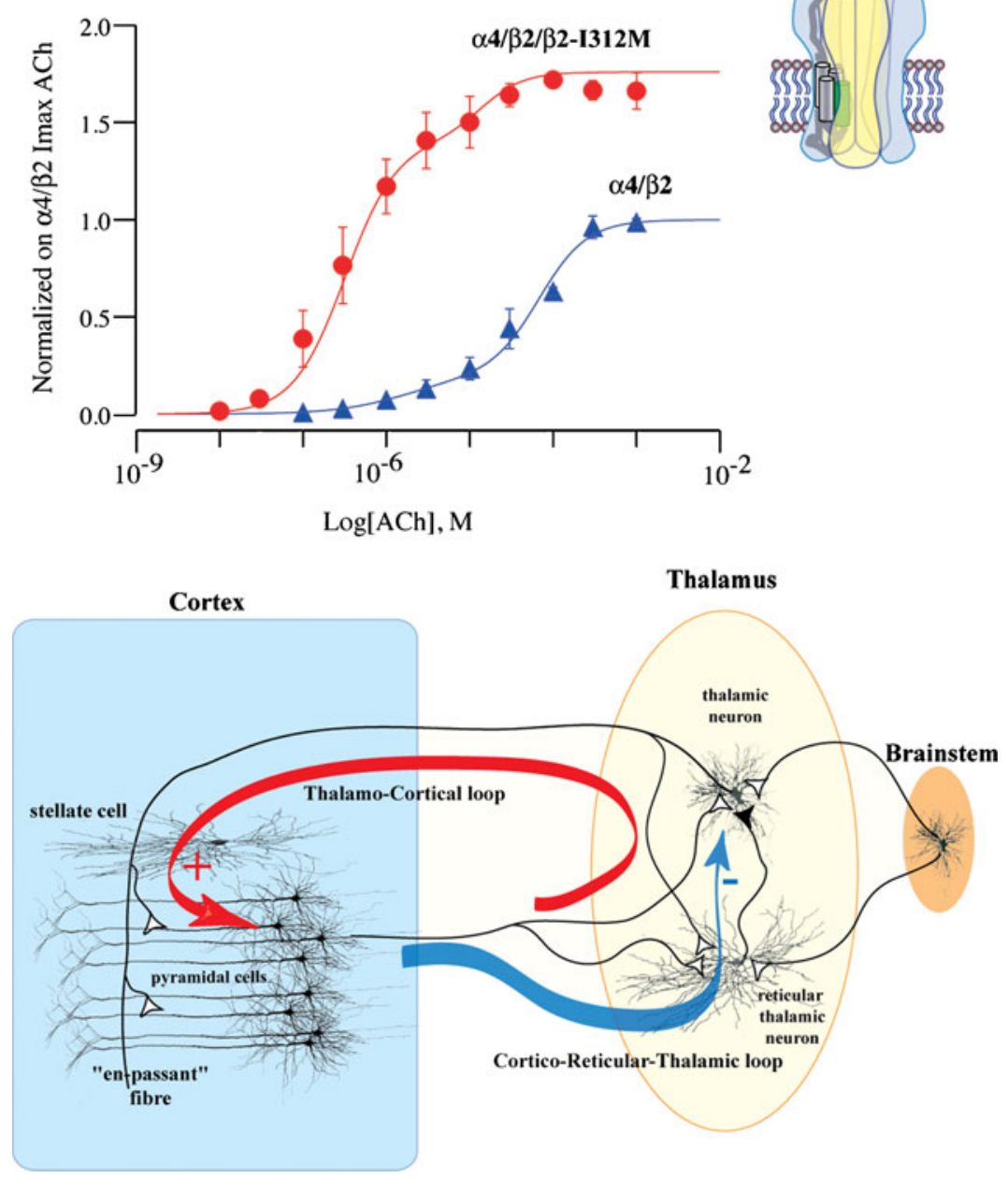

Fig. 1 Autosomal dominant nocturnal frontal lobe epilepsy (ADNFLE)-associated modification of the nicotinic acetylcholine receptors (nAChRs) and their putative effects on the brain network. Upper panel: schematic representation of the receptor inserted into the membrane and typical effects caused by a mutation in the $\beta 2$ subunit on the concentration activation curve. Plot represented in this figure illustrates the relationship between the acetylcholine concentration and the amplitude of the evoked response for the control $\alpha 4 \beta 2$ receptor (blue triangles). Curve through the data point is the best fit obtained with two Hill equations with a high and a low affinity. Note the wide

control $\beta 2$ confirmed the gain of acetylcholine sensitivity caused by this mutation together with additional modifications in the receptor properties such as a gain of sensitivity to nicotine and in the carbamazepine blockade [29].

From a statistical point of view, it can be predicted that mutations in $\alpha$ and $\beta$ nAChR genes should have a significant probability and might be found in the population with a higher frequency of occurrence than those reported up to now. In this respect, however, it should be remembered that the biological marker, which allowed the identification of the known mutants, is the clinical phenotype of epilepsy. Mutations causing more subtle range for the acetylcholine concentrations. Effects caused by a point mutation in the $\beta 2$ subunit are illustrated by the red dot and curve (for details, see Bertrand et al. [4]). Lower panel: representation of the main components of the brain network that are supposed to contribute to ADNFLE. Arrows illustrate the interactions between the thalamus and the cortex with a negative feedback from the cortical thalamic projections. The current hypothesis is that the gain of function of $\alpha 4 \beta 2 \mathrm{nAChR}$ variants causes an unbalance of excitation versus inhibition which in turn promotes seizure triggering

effects in the receptor functions and therefore having lower impact on the neuronal network properties have not yet been identified in correlation with a clinical phenotype.

\section{Mutations outside the channel domain}

Looking at the distribution pattern of ADNFLE mutations, it becomes obvious that they so far have only been found in the rather small parts of the CHRNA4 and CHRNB2 subunits that contribute to the channel domain. However, this does not necessarily implicate that mutations found in 
future studies must also be restricted to this segment of the protein. The pathophysiological effect of a given nAChR mutation most likely depends more on the functional pathways within the receptor to which the affected amino acid participates in than on the actual localization of the mutation within the subunit. This is possible because contiguous structures link certain amino acids from the channel domain to their functional counterparts within the binding sites. The fact that the three principal mechanisms of cys-loop receptors, i.e., agonist binding, coupling of binding to gating, and gating, are linked by functional pathways renders it possible that epilepsy might be caused by mutations at amino acids outside the channel domains of CHRNA4 and CHRNB2. Based on the current knowledge of ADNFLE, such mutation should however result in a gain of function, and it is possible to speculate where mutation causing a gain of function might occur. First, and as noted by Taly and Changeux [50], mutations affecting receptor function are most likely to be found at the interface between subunits. Second, mutations in the binding site causing a gain of function must render the receptor more sensitive to ACh. Structure function studies have already shown that mutations in the $\mathrm{N}$-terminal domain of the $\alpha$ or $\beta$ subunits can yield functional receptors that display a higher sensitivity to ACh (D.B. personal data).

Screening for ADNFLE mutations should therefore not be restricted to the transmembrane domain but must be extended to the entire gene structure. However, it should be kept in mind that mutations in different parts of nAChRs might not cause necessarily exactly the same disorder but some variant of it. It is possible that CHRNA4 or CHRNB2 not only harbor mutations that result in a gain but also ones that cause a loss of function. It can be expected that the clinical phenotypes caused by the latter class of mutations, if they indeed exist, would not resemble ADNFLE but may cause distinct type of neurological disorders. Further work attempting to correlate receptor mutations with the physiological modifications they cause and their possible association with clinical phenotypes is therefore needed.

\section{From receptor dysfunction to neuronal network alteration}

While functional studies carried out in expression systems allow detailed physiological and pharmacological investigations, they can only provide predictions on the effects on neuronal networks, and it is therefore indispensable to examine the outcomes in animal models. Although several laboratories attempted to produce mice models carrying ADNFLE-like mutations, few genetically engineered mice resulted in useful systems. Mice harboring a gain of function mutation in L9' of the $\alpha 4$ subunit (equivalent to
L247T initially described for the $\alpha 7$ subunit [44]) display a higher sensitivity to nicotine-induced seizures than wild type mice [16, 17]. Subsequently, knock-in mice were engineered that carried the equivalents of human $\alpha 4$ ADNFLE mutations [31, 34, 51]. These mice presented abnormal electroencephalography patterns, but only some of them presented spontaneous seizures. The seizures could be blocked by picrotoxin, an open channel blocker of the $\mathrm{GABA}_{\mathrm{A}}$ receptors, and it was therefore suggested that hypersynchronization was mediated by GABAergic neurons [31]. In agreement with this hypothesis, it had been documented that in the hippocampus, nAChRs can modulate the release of the neurotransmitter GABA and could interact with interneurons $[31,36]$.

Introduction of the $\alpha 4$ mutation $\mathrm{S} 252 \mathrm{~L}$ in a transgenic rat model (referred as S248L) yielded, however, different conclusions. Contrary to mice, this rat model shows spontaneous epileptic seizures on arousal during slow-wave-sleep (SWS) similar to those observed in ADNFLE patients. Moreover, electrophysiological recordings carried out in brain slices revealed two major abnormalities with a reduced GABAergic synaptic and extrasynaptic transmission and abnormal glutamate release during SWS [57]. The differences observed between the mice and rat models suggest that further analyses in different mammalian species is necessary before reaching a conclusion about the multiple brain dysfunctions caused by these point mutations and how they cause epileptic seizures. A schematic representation of the brain networks thought to participate in the epileptiform activity is represented in the lower panel of Fig. 1.

Functional heteromeric receptors comprise of at least two subunit subtypes such as in $\alpha 4 \beta 2$ nAChRs. As receptors result from the assembly of five subunits, a single receptor protein comprises a non-equivalent ratio of $\alpha$ and $\beta$ subunits. Differences in ratio between $\alpha$ and $\beta$ have been proposed to be at the origin of the high and low affinities observed for the $\alpha 4 \beta 2$ nAChRs revealing the complexity of these receptors even for two subunit compositions. Importantly, the number of possible combinations to form a functional receptor differ for $\alpha$ and $\beta$ subunits. Typically, while $\alpha 4$ can combine either with $\beta 2$ or $\beta 4$ subunits, the $\beta 2$ subunit can combine with $\alpha 2, \alpha 3, \alpha 4, \alpha 5, \alpha 6$, and putatively with the $\alpha 7$ subunit. This seems to suggest that mutations in the $\alpha 4$ subunit might affect a more restricted number of receptor subtypes than mutations in the $\beta 2$ subunit. However, mutations in both the $\alpha 4$ and the $\beta 2$ subunit cause the same epilepsy phenotype, which might suggests that mutations in $\beta 2$ only cause a neurological phenotype when co-assembled with $\alpha 4$. Another possible explanation would be that the epileptogenic effect only occurs when mutant $\mathrm{nAChRs}$ are expressed in certain brain structures in which the $\alpha 4 / \beta 2$ heteromeric receptor happens to be the main nAChR. 


\section{nAChRs and cognitive deficits}

Although limited in number, data obtained from the different families affected by ADNFLE reveal an association between certain mutations and cognitive impairment $[11,35]$. Examples are the CHRNA4 mutation S252F that co-segregates with mental retardation in two unrelated families or the CHRNB2 mutation I312M which was found in siblings that showed specific cognitive dysfunctions [4, $11,27,35]$. The neuronal nAChRs are known to have an important role in shaping synaptic connections and determining plasticity in brain areas involved in fundamental aspects of cognition. It would therefore not be surprising if future studies showed that the observed association between certain ADNFLE mutations and cognitive defects is indeed a causative one. Until then, one has also to keep in mind that the cognitive impairments might result from the epileptic seizures themselves, and might therefore be only indirectly related to the nAChR mutations. However, such an indirect effect would hardly explain the observation that other ADNFLE mutations, such as CHRNA4 S248F, are almost never associated with cognitive defects. The latter phenomena would be much easier to explain by a mutationspecific effect. Such an effect would be likely to depend somehow on the specific position of the mutated amino acid within the receptor because neighboring mutations such as $\mathrm{S} 248 \mathrm{~F}$ and $\mathrm{S} 252 \mathrm{~F}$ are discordant with respect to their associations to cognitive defects. The mutationspecific hypothesis is further supported by the so far only known CHRNA2 mutation [2]. Experimental evidences obtained from animal studies indicate that $\beta 2$ containing receptors participate in the signal elaboration in the prefrontal cortex as well as other cortical areas [43]. As $\alpha 2$ subunits are largely expressed in the prefrontal cortex of higher mammals, it was proposed that receptors containing $\alpha 2$ might participate in cognitive processing [24, 25]. However, the so far only known family harboring an epilepsy-causing mutation in CHRNA2 does not display major cognitive impairments. This could suggest either that the epilepsy in this family is too benign to interfere with higher brain functions or that it is just this specific $\alpha 2$ mutation that does not markedly affect cognitive processes.

Although all mutations identified so far result in a receptor gain of function, it is also intriguing to speculate about the putative effects of the above-discussed hypothetic mutations that cause reduced receptor activity. Animal experiments carried out with $\alpha 4 \beta 2$ antagonists such as dihydro-beta-erythroidine or mecamylamine clearly indicate that inhibition of this receptor subtype is detrimental. This observation is in good correlation with the clinical data observed in neurodegenerative disorders such as Alzheimer where a preferential loss of the nicotinic receptor was observed during the course of this disease. Loss of function mutations are therefore at least as likely to interfere with cognitive processes as gain of function mutations are suspected to do.

While further work is required before obtaining a clear relationship between $\mathrm{nAChR}$ and cognitive functions, these studies underline the important role of nAChRs sequence variants and prompt for more detailed studies of rare mutations, common genetic variants, and cognitive capacities.

Acknowledgments This work was supported by the Swiss National Science Foundation to DB (3100A0-101787/2) and by the DFG (STE16511-2, BE 3834/1-2) to OKS and to DB.

\section{References}

1. Albuquerque EX, Pereira EF, Alkondon M, Rogers SW (2009) Mammalian nicotinic acetylcholine receptors: from structure to function. Physiol Rev 89:73-120

2. Aridon P, Marini C, Di Resta C, Brilli E et al (2006) Increased sensitivity of the neuronal nicotinic receptor alpha 2 subunit causes familial epilepsy with nocturnal wandering and ictal fear. Am J Hum Genet 79:342-350

3. Ballivet M, Nef P, Couturier S, Rungger D et al (1988) Electrophysiology of a chick neuronal nicotinic acetylcholine receptor expressed in Xenopus oocytes after cDNA injection. Neuron 1:847-852

4. Bertrand D, Elmslie F, Hughes E, Trounce J et al (2005) The CHRNB2 mutation I312M is associated with epilepsy and distinct memory deficits. Neurobiol Dis 20:799-804

5. Bertrand D, Galzi JL, Devillers-Thiéry A, Bertrand S, Changeux JP (1993) Mutations at two distinct sites within the channel domain M2 alter calcium permeability of neuronal alpha 7 nicotinic receptor. Proc Natl Acad Sci U S A 90:6971-6975

6. Bertrand D, Picard F, Le Hellard S, Weiland S et al (2002) How mutations in the nAChRs can cause ADNFLE epilepsy. Epilepsia 43(Suppl 5):112-122

7. Bertrand S, Weiland S, Berkovic SF, Steinlein OK, Bertrand D (1998) Properties of neuronal nicotinic acetylcholine receptor mutants from humans suffering from autosomal dominant nocturnal frontal lobe epilepsy. Br J Pharmacol 125:751-760

8. Bocquet N, Nury H, Baaden M, Le Poupon C et al (2009) X-ray structure of a pentameric ligand-gated ion channel in an apparently open conformation. Nature 457:111-114

9. Buisson B, Bertrand D (2001) Chronic exposure to nicotine upregulates the human (alpha)4((beta)2 nicotinic acetylcholine receptor function. J Neurosci 21:1819-1829

10. Buisson B, Gopalakrishnan M, Arneric SP, Sullivan JP, Bertrand D (1996) Human alpha4beta2 neuronal nicotinic acetylcholine receptor in HEK 293 cells: a patch-clamp study. J Neurosci 16:7880-7891

11. Cho YW, Motamedi GK, Laufenberg I, Sohn SI et al (2003) A Korean kindred with autosomal dominant nocturnal frontal lobe epilepsy and mental retardation. Arch Neurol 60:1625-1632

12. Cooper E, Couturier S, Ballivet M (1991) Pentameric structure and subunit stoichiometry of a neuronal nicotinic acetylcholine receptor. Nature 350:235-238

13. Dani JA, Bertrand D (2007) Nicotinic acetylcholine receptors and nicotinic cholinergic mechanisms of the central nervous system. Annu Rev Pharmacol Toxicol 47:699-729

14. De Fusco M, Becchetti A, Patrignani A, Annesi G et al (2000) The nicotinic receptor beta 2 subunit is mutant in nocturnal frontal lobe epilepsy. Nat Genet 26:275-276 
15. Figl A, Viseshakul N, Shafaee N, Forsayeth J, Cohen BN (1998) Two mutations linked to nocturnal frontal lobe epilepsy cause usedependent potentiation of the nicotinic ACh response. J Physiol 513(Pt 3):655-670

16. Fonck C, Cohen BN, Nashmi R, Whiteaker P et al (2005) Novel seizure phenotype and sleep disruptions in knock-in mice with hypersensitive alpha $4 *$ nicotinic receptors. J Neurosci 25:1139611411

17. Fonck C, Nashmi R, Deshpande P, Damaj MI et al (2003) Increased sensitivity to agonist-induced seizures, straub tail, and hippocampal theta rhythm in knock-in mice carrying hypersensitive alpha 4 nicotinic receptors. J Neurosci 23:2582-2590

18. Forster I, Bertrand D (1995) Inward rectification of neuronal nicotinic acetylcholine receptors investigated by using the homomeric alpha 7 receptor. Proc Biol Sci 260:139-148

19. Fucile S (2004) Ca2+ permeability of nicotinic acetylcholine receptors. Cell Calcium 35:1-8

20. Fucile S, Sucapane A, Eusebi F (2005) $\mathrm{Ca} 2+$ permeability of nicotinic acetylcholine receptors from rat dorsal root ganglion neurones. J Physiol 565:219-228

21. Galzi JL, Devillers-Thiéry A, Hussy N, Bertrand S et al (1992) Mutations in the channel domain of a neuronal nicotinic receptor convert ion selectivity from cationic to anionic. Nature 359:500-505

22. Gilbert D, Lecchi M, Arnaudeau S, Bertrand D, Demaurex N (2009) Local and global calcium signals associated with the opening of neuronal alpha7 nicotinic acetylcholine receptors. Cell Calcium 45:198-207

23. Gopalakrishnan M, Buisson B, Touma E, Giordano T et al (1995) Stable expression and pharmacological properties of the human alpha 7 nicotinic acetylcholine receptor. Eur J Pharmacol 290:237-246

24. Han ZY, Le Novère N, Zoli M, Hill JA et al (2000) Localization of nAChR subunit mRNAs in the brain of Macaca mulatta. Eur J NeuroSci 12:3664-3674

25. Han ZY, Zoli M, Cardona A, Bourgeois JP et al (2003) Localization of $[3 \mathrm{H}]$ nicotine, $[3 \mathrm{H}]$ cytisine, $[3 \mathrm{H}]$ epibatidine, and [125I]alpha-bungarotoxin binding sites in the brain of Macaca mulatta. J Comp Neurol 461:49-60

26. Hilf RJ, Dutzler R (2009) A prokaryotic perspective on pentameric ligand-gated ion channel structure. Curr Opin Struct Biol 19:418-424

27. Hirose S, Iwata H, Akiyoshi H, Kobayashi K et al (1999) A novel mutation of CHRNA4 responsible for autosomal dominant nocturnal frontal lobe epilepsy. Neurology 53:1749-1753

28. Hoda JC, Gu W, Friedli M, Phillips HA et al (2008) Human nocturnal frontal lobe epilepsy: pharmocogenomic profiles of pathogenic nicotinic acetylcholine receptor beta-subunit mutations outside the ion channel pore. Mol Pharmacol 74:379-391

29. Hoda JC, Wanischeck M, Bertrand D, Steinlein OK (2009) Pleiotropic functional effects of the first epilepsy-associated mutation in the human CHRNA2 gene. FEBS Lett 583:1599-1604

30. Itier V, Bertrand D (2002) Mutations of the neuronal nicotinic acetylcholine receptors and their association with ADNFLE. Neurophysiol Clin 32:99-107

31. Klaassen A, Glykys J, Maguire J, Labarca C et al (2006) Seizures and enhanced cortical GABAergic inhibition in two mouse models of human autosomal dominant nocturnal frontal lobe epilepsy. Proc Natl Acad Sci U S A 103:19152-19157

32. Kuryatov A, Gerzanich V, Nelson M, Olale F, Lindstrom J (1997) Mutation causing autosomal dominant nocturnal frontal lobe epilepsy alters $\mathrm{Ca} 2+$ permeability, conductance, and gating of human alpha4beta2 nicotinic acetylcholine receptors. J Neurosci 17:9035-9047

33. Leniger T, Kananura C, Hufnagel A, Bertrand S et al (2003) A new Chrna4 mutation with low penetrance in nocturnal frontal lobe epilepsy. Epilepsia 44:981-985
34. Lipovsek M, Plazas P, Savino J, Klaassen A et al (2008) Properties of mutated murine alpha4beta2 nicotinic receptors linked to partial epilepsy. Neurosci Lett 434:165-169

35. Magnusson A, Stordal E, Brodtkorb E, Steinlein O (2003) Schizophrenia, psychotic illness and other psychiatric symptoms in families with autosomal dominant nocturnal frontal lobe epilepsy caused by different mutations. Psychiatr Genet 13: 91-95

36. Mann EO, Mody I (2008) The multifaceted role of inhibition in epilepsy: seizure-genesis through excessive GABAergic inhibition in autosomal dominant nocturnal frontal lobe epilepsy. Curr Opin Neurol 21:155-160

37. McLellan A, Phillips HA, Rittey C, Kirkpatrick M et al (2003) Phenotypic comparison of two Scottish families with mutations in different genes causing autosomal dominant nocturnal frontal lobe epilepsy. Epilepsia 44:613-617

38. Moroni M, Bermudez I (2006) Stoichiometry and pharmacology of two human alpha4beta2 nicotinic receptor types. J Mol Neurosci 30:95-96

39. Patrick J, Boulter J, Deneris E, Wada K et al (1989) Structure and function of neuronal nicotinic acetylcholine receptors deduced from cDNA clones. Prog Brain Res 79:27-33

40. Phillips HA, Favre I, Kirkpatrick M, Zuberi SM et al (2001) CHRNB2 is the second acetylcholine receptor subunit associated with autosomal dominant nocturnal frontal lobe epilepsy. Am J Hum Genet 68:225-231

41. Phillips HA, Marini C, Scheffer IE, Sutherland GR et al (2000) A de novo mutation in sporadic nocturnal frontal lobe epilepsy. Ann Neurol 48:264-267

42. Picard F, Bertrand S, Steinlein OK, Bertrand D (1999) Mutated nicotinic receptors responsible for autosomal dominant nocturnal frontal lobe epilepsy are more sensitive to carbamazepine. Epilepsia 40:1198-1209

43. Poorthuis RB, Goriounova NA, Couey JJ, Mansvelder HD (2009) Nicotinic actions on neuronal networks for cognition: general principles and long-term consequences. Biochem Pharmacol 78:668-676

44. Revah F, Bertrand D, Galzi JL, Devillers-Thiéry A et al (1991) Mutations in the channel domain alter desensitization of a neuronal nicotinic receptor. Nature 353:846-849

45. Sands SB, Barish ME (1991) Calcium permeability of neuronal nicotinic acetylcholine receptor channels in PC12 cells. Brain Res 560:38-42

46. Séguéla P, Wadiche J, Dineley-Miller K, Dani JA, Patrick JW (1993) Molecular cloning, functional properties, and distribution of rat brain alpha 7: a nicotinic cation channel highly permeable to calcium. J Neurosci 13:596-604

47. Steinlein OK, Bertrand D (2008) Neuronal nicotinic acetylcholine receptors: from the genetic analysis to neurological diseases. Biochem Pharmacol 76:1175-1183

48. Steinlein OK, Magnusson A, Stoodt J, Bertrand S et al (1997) An insertion mutation of the CHRNA4 gene in a family with autosomal dominant nocturnal frontal lobe epilepsy. Hum Mol Genet 6:943-947

49. Steinlein OK, Mulley JC, Propping P, Wallace RH et al (1995) A missense mutation in the neuronal nicotinic acetylcholine receptor alpha 4 subunit is associated with autosomal dominant nocturnal frontal lobe epilepsy. Nat Genet 11:201-203

50. Taly A, Changeux JP (2008) Functional organization and conformational dynamics of the nicotinic receptor: a plausible structural interpretation of myasthenic mutations. Ann N Y Acad Sci 1132:42-52

51. Teper Y, Whyte D, Cahir E, Lester HA et al (2007) Nicotineinduced dystonic arousal complex in a mouse line harboring a human autosomal-dominant nocturnal frontal lobe epilepsy mutation. J Neurosci 27:10128-10142 
52. Unwin N (2005) Refined structure of the nicotinic acetylcholine receptor at 4A resolution. J Mol Biol 346:967-989

53. Weiland S, Witzemann V, Villarroel A, Propping P, Steinlein O (1996) An amino acid exchange in the second transmembrane segment of a neuronal nicotinic receptor causes partial epilepsy by altering its desensitization kinetics. FEBS Lett 398:91-96

54. Williams BM, Temburni MK, Levey MS, Bertrand S et al (1998) The long internal loop of the alpha 3 subunit targets nAChRs to subdomains within individual synapses on neurons in vivo. Nat Neurosci 1:557-562
55. Xu J, Zhu Y, Heinemann SF (2006) Identification of sequence motifs that target neuronal nicotinic receptors to dendrites and axons. J Neurosci 26:9780-9793

56. Zhou Y, Nelson ME, Kuryatov A, Choi C et al (2003) Human alpha4beta2 acetylcholine receptors formed from linked subunits. J Neurosci 23:9004-9015

57. Zhu G, Okada M, Yoshida S, Ueno S et al (2008) Rats harboring S284L Chrna4 mutation show attenuation of synaptic and extrasynaptic GABAergic transmission and exhibit the nocturnal frontal lobe epilepsy phenotype. J Neurosci 28:12465-12476 